\title{
Suppression of Oscillation of a Certain Two-Mass System with the Help of the Generalized Gauss Principle
}

\author{
Kamilla Maratovna Fazlyeva, Timofei Sergeevich Shugailo, Mikhail Petrovich Yushkov
}

Department of Theoretical and Applied Mechanics, St. Petersburg University, St. Petersburg, Russia

Email address:

flora666@ya.ru (K. M. Fazlyeva), shugaylotis@gmail.com (T. S. Shugaylo), yushkovmp@mail.ru (M. P. Yushkov)

\section{To cite this article:}

Kamilla Maratovna Fazlyeva, Timofei Sergeevich Shugailo, Mikhail Petrovich Yushkov. Suppression of Oscillation of a Certain Two-Mass System with the Help of the Generalized Gauss Principle. International Journal of Mechanical Engineering and Applications.

Vol. 5, No. 3, 2017, pp. 129-135. doi: 10.11648/j.ijmea.20170503.11

Received: February 1, 2017; Accepted: May 6, 2017; Published: May 10, 2017

\begin{abstract}
The paper studies the suppression of oscillation of a certain two-mass system when it is transferred from the initial state of rest to the given state of rest during a time interval prescribed. The problem is solved by the two methods: the Pontryagin maximum principle (first method) and the generalized Gauss principle (second method). Computational results are presented and the solutions are compared to each other. When the time of motion is short the both methods give practically the same results, but when the time of motion is long the results differ widely. If the time of motion is long then the second method is more preferable than the first one, since the control obtained by the second method sways the mechanical system less than the control obtained by the classical approach. This can be explained by the fact that the first method contains the control including harmonics with the natural frequency of the system, and this seeks to put the system into resonance. In contrast to this, in the second method the control is sought in the form of time polynomial that provides relatively smooth motion of the system. It is noted that the first method always finds the control with jumps at the beginning and at the end of motion. The second method also gives the same jumps when the time of motion is short, but when the time of motion is long the similar jumps vanish when one uses the generalized Gauss principle.
\end{abstract}

Keywords: Pontryagin Maximum Principle, Generalized Gauss Principle, Control Force, Suppression of Oscillation

\section{Introduction}

One of the most important problems of the control theory is the problem of finding the optimal force that transfers a mechanical system during the time required from a phase state for which the generalized coordinates and velocities are given to the final phase state with the generalized coordinates and velocities prescribed. If the final phase state is the state of rest, then such a problem is called the problem of suppression of oscillation.

The classical methods applied to solving similar problems are based, first of all, on the Pontryagin maximum principle [1] and on the dynamical programming [2] developed by R. Bellman and his scholars. Academician N. N. Moiseev [3] advises to use the local extremum method by F. L. Chernous'ko [4].

This paper uses a new method basing on the application of the generalized Gauss principle [5] to solving the problem of suppression of oscillation of a two-mass system with a spring.
Its results will be compared to the application of the Pontryagin maximum principle. Solving the problem formulated continues the cycle of investigations performed earlier at the Department of Theoretical and Applied Mechanics of Saint Petersburg State University (for example, see works [6-10]). The first work in this cycle was the paper [11].

\section{Equations of Motion}

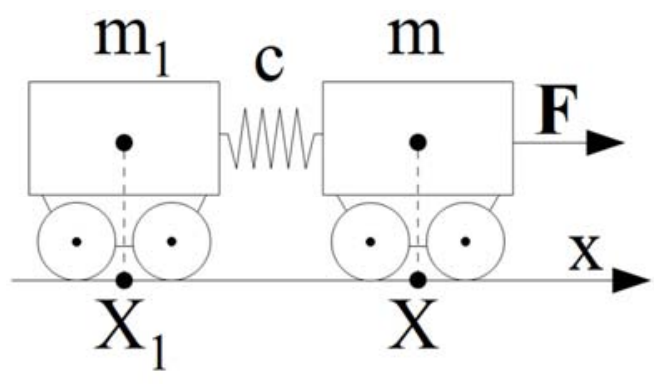

Figure 1. Two-mass system. 
Consider the problem of horizontal motion of two particles with masses $m$ and $m_{1}$ connected to each other by the spring of stiffness $c$ along the $x$-axis (Figure 1). The motion of the mechanical system is realized under the action of the control horizontal force $\mathrm{F}$, applied to the right mass $m$. It is required to find such a control force $F$ that the system could be moved from the initial state of rest by the distance $S$ to a new state of rest during the time $\tilde{T}$.

Denote the dimensional coordinates of the masses by $X$ and $X_{1}$. Then the differential equations of motion of the mechanical system are

$$
\begin{gathered}
m \ddot{X}+c\left(X-X_{1}\right)=F, \\
m_{1} \ddot{X}_{1}+c\left(X_{1}-X\right)=0 .
\end{gathered}
$$

In the problem formulated the following boundary conditions should be fulfilled:

$$
\begin{array}{llll}
X(0)=0, & X_{1}(0)=0, & \dot{X}(0)=0, & \dot{X}_{1}(0)=0 \\
X(\tilde{T})=S, & X_{1}(\tilde{T})=S, & \dot{X}(\tilde{T})=0, & \dot{X}_{1}(\tilde{T})=0 .
\end{array}
$$

Note that two equations (1) contain three unknown time functions $X, X_{1}, F$. In monograph [12] this indeterminacy is eliminated by means of the condition of minimization of the functional

$$
J=\int_{0}^{\tilde{T}} F^{2}(t) d t
$$

For the sake of convenience in further investigations, we shall write the equations of motion of the system in principal coordinates [13]. Seeking the solution of the homogeneous system corresponding to nonhomogeneous system of differential equations (1) in the form

$$
\begin{aligned}
X & =H \sin (\Omega t+\alpha), \\
X_{1} & =H_{1} \sin (\Omega t+\alpha),
\end{aligned}
$$

we get the system of homogeneous linear algebraic equations

$$
\begin{gathered}
\left(\frac{c}{m}-\Omega^{2}\right) H-\frac{c}{m} H_{1}=0, \\
-\frac{c}{m_{1}} H+\left(\frac{c}{m_{1}}-\Omega^{2}\right) H_{1}=0 .
\end{gathered}
$$

Setting the determinant of the system equal to zero and writing it in the extended form, we obtain a characteristic equation, from the roots of which the dimensional natural frequencies are found

$$
\Omega_{0}=0, \quad \Omega_{1}=\sqrt{\frac{c\left(m+m_{1}\right)}{m m_{1}}} .
$$

For each of the frequencies (5) from system of equations (4) we arrive at the normal modes of vibration

$$
(1,1), \quad\left(1,-\frac{m}{m_{1}}\right) \text {. }
$$

Basing on the normal modes (6), we construct the general solution of the homogeneous system of differential equations in question

$$
\begin{gathered}
X=D_{0}+D_{1} t+D_{2} \sin \left(\Omega_{1} t+\alpha\right), \\
X_{1}=D_{0}+D_{1} t-D_{2} \frac{m}{m_{1}} \sin \left(\Omega_{1} t+\alpha\right) .
\end{gathered}
$$

Supposing that the principal coordinates $y_{0}$ and $y_{1}$ change according to the laws

$$
\begin{gathered}
y_{0}=D_{0}+D_{1} t, \\
y_{1}=D_{2} \sin \left(\Omega_{1} t+\alpha\right),
\end{gathered}
$$

from solution (7) we get the coordinate transformation

$$
X=y_{0}+y_{1}, \quad X_{1}=y_{0}-\frac{m}{m_{1}} y_{1},
$$

which makes it possible to write the system of the Lagrange equations of second kind in principal dimensional coordinates $y_{0}$ and $y_{1}$ in the form of an independent system of two equations

$$
M \ddot{y_{0}}=F, \quad \ddot{y}_{1}+\frac{c M}{m m_{1}} y_{1}=\frac{F m_{1}}{M m}, \quad M=m+m_{1} .
$$

Note that according to transformation (8)

$$
y_{0}=\frac{m X+m_{1} X_{1}}{M},
$$

that is why the first equation in (9) represents the principle of motion of centre of mass of the mechanical system in question.

Introduce the dimensionless quantities

$$
\begin{array}{lc}
u=\frac{F m_{1}^{2}}{c M^{2} S}, & x_{0}=\frac{m_{1}}{m} \frac{y_{0}}{S}, \quad x_{1}=\frac{y_{1}}{S}, \\
\tau=\Omega_{1} t, \quad T=\Omega_{1} \tilde{T}, \quad \omega_{1}=\frac{\Omega_{1}}{\Omega_{1}}=1 .
\end{array}
$$

Using notation (10), formulas (9), (2), (3) appear as

$$
\begin{gathered}
x_{0}^{\prime \prime}=u, \quad x_{1}^{\prime \prime}+x_{1}=u, \\
x_{0}(0)=0, \quad x_{1}(0)=0, \quad x_{0}^{\prime}(0)=0, \quad x_{1}^{\prime}(0)=0, \\
x_{0}(T)=1, \quad x_{1}(T)=1, \quad x_{0}^{\prime}(T)=0, \quad x_{1}^{\prime}(T)=0, \\
J=\int_{0}^{T} u^{2}(\tau) d \tau .
\end{gathered}
$$

Here the prime denotes differentiation in dimensionless time $\tau$. Since a linear problem is solved, without loss of generality we assume that the masses move by the dimensionless distance equal to unit. Besides this, we succeeded in obtaining the same nonhomogeneity $u$ due to linear transformation of one of the principal coordinates in (10).

\section{Solving the Problem by the Pontryagin Maximum Principle}

We'll seek the optimal control with the help of minimization of functional (14) applying the Pontryagin maximum principle [1]. According to the general theory we write equations (11) in the form of system of differential equations of the first order 


$$
\begin{gathered}
z_{k}^{\prime}=f_{k}, \quad k=\overline{1,4}, \\
f_{1}=z_{2}, \quad f_{2}=u, \quad f_{3}=z_{4}, \quad f_{4}=u-z_{3},
\end{gathered}
$$

and compose the Hamilton-Pontryagin function

$$
H=-u^{2}+\lambda_{1} z_{2}+\lambda_{2} u+\lambda_{3} z_{4}+\lambda_{4}\left(u-z_{3}\right) .
$$

The system of equations for finding the Lagrange multipliers $\lambda_{k}$ and control $u$

$$
\lambda_{k}^{\prime}=-\frac{\partial H}{\partial z_{k}}, \quad k=\overline{1,4}, \quad \frac{\partial H}{\partial u}=0,
$$

in this case appears as

$$
\lambda_{2}^{\prime \prime}=0, \quad \lambda_{4}^{\prime \prime}+\lambda_{4}=0, \quad 2 u=\lambda_{2}+\lambda_{4} .
$$

System (11) implies that the control has the form

$$
u(\tau)=C_{1}+C_{2} \tau+C_{3} \sin (\tau)+C_{4} \cos (\tau),
$$

where $C_{k}, k=\overline{1,4}$, are arbitrary constants.

The partial solutions of equations (11) satisfying the zero initial conditions (in this case boundary conditions (12)) can be represented by the Duhamel integrals

$$
\begin{gathered}
x_{0}=\int_{0}^{\tau} u\left(\tau_{1}\right)\left(\tau-\tau_{1}\right) d \tau_{1}, \\
x_{1}=\int_{0}^{\tau} u\left(\tau_{1}\right) \sin \left(\tau-\tau_{1}\right) d \tau_{1} .
\end{gathered}
$$

Substituting (16) into integrals (17) and satisfying boundary conditions (13), we get a system of linear inhomogeneous algebraic equations with respect to the sought constants $C_{k}, k=\overline{1,4}$. For the four cases of motion when $T=2 \pi ; 8 \pi ; 16 \pi ; 32 \pi$ we have

$$
\begin{aligned}
& T=2 \pi: \begin{array}{cc}
C_{1}=0.387637, & C_{2}=-0.123389, \\
C_{3}=0, & C_{4}=-0.246777,
\end{array} \\
& T=8 \pi: \begin{array}{cc}
C_{1}=0.009874, & C_{2}=-0.000786, \\
C_{3}=0, & C_{4}=-0.0015715,
\end{array} \\
& T=16 \pi: \begin{array}{cc}
C_{1}=0.002397, & C_{2}=-0.000095, \\
C_{3}=0, & C_{4}=-0.00019,
\end{array} \\
& T=32 \pi: \begin{array}{cc}
C_{1}=0.000595, & C_{2}=-0.000012, \\
C_{3}=0, & C_{4}=-0.000024 .
\end{array}
\end{aligned}
$$

The computational results are presented in Figures 2, 3, 4 and 5 by dashed lines.

\section{Relationship Between the Solution and Nonholonomic Mechanics}

Now consider the solution obtained by the Pontryagin maximum principle minimizing functional (14) from a totally new viewpoint. To this end, note that the control (16) obtained by the Pontryagin maximum principle is the solution of differential equation

$$
\frac{d^{2}}{d \tau^{2}}\left(\frac{d^{2}}{d \tau^{2}}+\omega_{1}^{2}\right) u=0, \quad \omega_{1}^{2}=1
$$

Returning in equation (18) from dimensionless variables to dimensional ones, we get

$$
\frac{d^{2}}{d t^{2}}\left(\frac{d^{2}}{d t^{2}}+\Omega_{1}^{2}\right) F=0 .
$$

If we substitute the expression for $F$ taken from the first equation of system (1) into (19) then we have

$$
\begin{gathered}
m \frac{d^{6} X}{d t^{6}}+\left(c+m \Omega_{1}^{2}\right) \frac{d^{4} X}{d t^{4}}-c \frac{d^{4} X_{1}}{d t^{4}}+ \\
+c \Omega_{1}^{2} \frac{d^{2} X}{d t^{2}}-c \Omega_{1}^{2} \frac{d^{2} X_{1}}{d t^{2}}=0 .
\end{gathered}
$$

Differential equation (20) can be considered as a nonholonomic sixth-order(!) constraint that is continuously realized when moving under the action of the control obtained by the minimization of functional (3). Thus, for the control obtained by the Pontryagin maximum principle the nonholonomic high-order constraint is continuously realized, and in this case one can try to solve the control problem formulated with the help of the theory of motion of nonholonomic high-order constraint systems developed in monograph [14]. This fact suggests that one should apply the generalized Gauss principle typical for the theory of motion of nonholonomic high-order constraint systems. Let us turn our attention to this principle.

\section{The Gauss Principle and the Generalized GAUSS Principle}

Let the kinetic energy $T$ of a mechanical system in curvilinear coordinates $q=\left(q^{1}, \ldots, q^{s}\right)$ has the form

$$
\begin{gathered}
T=T^{(2)}+T^{(1)}+T^{(0)}= \\
=\frac{M}{2} g_{\sigma \tau} \dot{q}^{\sigma} \dot{q}^{\tau}+M g_{0 \alpha} \dot{q}^{\alpha}+\frac{M}{2} g_{00}, \\
\sigma, \tau=\overline{1, s}, \alpha=\overline{0, s}, q^{0}=t, \dot{q^{0}}=1 .
\end{gathered}
$$

Here $M$ is the mass of the whole system. Introduce into consideration the differentiable manifold of all positions of the mechanical system which it can have at the given time moment $t$. Then the Lagrange equations of the second kind in the tangent space [15] constructed for the moment $t$ in the distinguished point $q=\left(q^{1}, \ldots, q^{s}\right)$ can be represented as a vector equality similar to the Newton second law:

$$
M \vec{W}=\vec{Y} .
$$

Here the acceleration of the system $\vec{W}$ and the vector of active forces $\vec{Y}$ are

$$
\begin{gathered}
\vec{W}=\frac{1}{M}\left(\frac{d}{d t} \frac{\partial T}{\partial \dot{q}^{\sigma}}-\frac{\partial T}{\partial q^{\sigma}}\right) \vec{e}^{\sigma}, \\
\vec{Y}=Q_{\sigma} \vec{e}^{\sigma} .
\end{gathered}
$$

Here the vectors $\vec{e}^{\sigma}, \sigma=\overline{1, s}$, are the vectors of reciprocal basis introduced in the tangent space. The main metric tensor $\left(g_{\sigma \tau}\right), \sigma, \tau=\overline{1, s}$, is given by the coefficients of a positive definite quadratic form $T^{(2)}$ (see formula (21)).

If we impose nonholonomic second-order constraints (to 
such a form can be reduced the both holonomic and nonholonomic constraints $f_{0}^{\kappa}(t, q)=0, f_{1}^{\kappa}(t, q, \dot{q})=0$ after differentiation in time)

$$
\begin{gathered}
f_{2}^{\kappa} \equiv \alpha_{2, \sigma}^{\kappa}(t, q, \dot{q}) \ddot{q}^{\sigma}+\alpha_{2,0}^{\kappa}(t, q, \dot{q})=0, \\
\kappa=\overline{1, k}
\end{gathered}
$$

on the motion of the system, then in the case of ideal constraints equation (22) appears as [14]

$$
\begin{gathered}
M \vec{W}=\vec{Y}+\Lambda_{\kappa} \vec{\varepsilon}^{l+\kappa}, \\
\vec{\varepsilon}^{l+\kappa}=\alpha_{2, \sigma}^{\kappa} \vec{e}^{\sigma}, l=s-k .
\end{gathered}
$$

The Lagrange multipliers $\Lambda_{\kappa}, \kappa=\overline{1, k}$, can be found as functions of $t, q, \dot{q}$ [14]. To equation (24) the Gauss principle corresponds ( $\delta^{\prime \prime}$ means that only the variables $\ddot{q}^{\sigma}$ are varied):

$$
\delta^{\prime \prime} Z=0, \quad Z=\frac{M}{2}\left(\vec{W}-\frac{\vec{Y}}{M}\right)^{2}
$$

Formulae (25) state that the reaction force $\vec{R}=M \vec{W}-\vec{Y}$ of ideal nonholonomic constraints (23) has the minimum value.

Suppose now that on the motion of the system the linear nonholonomic high-order constraints are imposed (the figures in degrees in brackets denote the order of derivatives in time)

$$
\begin{gathered}
f_{n+2}^{\kappa} \equiv \alpha_{n+2, \sigma}^{\kappa}\left(t, q, \dot{q}, \ldots, q^{(n+1)}\right) \ddot{q}^{\sigma(n+2)}+ \\
+\alpha_{n+2,0}^{\kappa}\left(t, q, \dot{q}, \ldots, q^{(n+1)}\right)=0 \\
\quad \kappa=\overline{1, k}, \sigma=\overline{1, s}, n \geq 1 .
\end{gathered}
$$

Then we can introduce the generalized Gauss principle

$$
\delta^{(n+2)} Z_{(n)}=0, \quad Z_{(n)}=\frac{M}{2}\left(\vec{W}^{(n)}-\frac{\vec{Y}^{(n)}}{M}\right)^{2} .
$$

The symbol $\delta^{(n+2)}$ in (27) means that only the $n+2^{\text {nd }}$ - order derivatives of generalized coordinates are varied. Formulae (27) imply the minimality of the vector

$$
\overrightarrow{\mathcal{R}} \equiv \vec{R}_{(n)}=M \frac{d^{n} \vec{W}}{d t^{n}}-\frac{d^{n} \vec{Y}}{d t^{n}} .
$$

Note that equations (26) should be regarded as the program of motion given in the form of additional system of differential equations that should be continuously realized in the process of motion of the mechanical system. That is why in nonholonomic mechanics the high-order constraints given in form (26) are usually called program constraints, the reaction force vector $\vec{R}$ formed by them plays the role of the control that provides realization of the given program. In its turn, vector (28) can be conventionally called "the reaction" of these high-order constraints. We also note that one should take into consideration [14] that due to the necessity of forming this reaction by technical devices the sought reaction is represented in the form $\vec{R}=\Lambda_{\kappa} \vec{b}^{\kappa}, \vec{b}^{\kappa}=b_{\sigma}^{\kappa} \vec{e}^{\sigma}$, where $\Lambda_{\kappa}$ are found along with the generalized coordinates as unknown time functions, and coefficients $b_{\sigma}^{\kappa}$ are usually given by the control system as constant values.

\section{Solving the Problem by Means of the Generalized Gauss Principle}

Thus, we have seen that the problem of interest can be considered as a mechanical problem on the motion of which the sixth-order nonholonomic constraint (20) is imposed. It was shown in monograph [14] that for finding the reaction force of such a constraint one should compose a fourth-order differential equation. So, if one considers constraint (20) as a certain program of motion that should be fulfilled by the mechanical system then the reaction force of this constraint turns out to be the control force providing realization of this program. That is why equation (20) with respect to the control can be interpreted as a differential equation with respect to the reaction force. In this case the reaction force can be represented as

$$
\vec{R}=u(t) \vec{b}, \vec{b}=\sum_{\sigma=1}^{2} b_{\sigma} \vec{e}^{\sigma} .
$$

When a sixth-order constraint is imposed, the Gauss principle states that "the reaction" of this constraint should be minimal, that is the quantity

$$
(\overrightarrow{\mathcal{R}})^{2} \equiv\left(\vec{R}_{(4)}\right)^{2}=\left(M \frac{d^{4} \vec{W}}{d t^{4}}-\frac{d^{4} \vec{Y}}{d t^{4}}\right)^{2}
$$

should be minimal.

From all possible nonholonomic sixth-order constraints we distinguish such a subset for the elements of which the quantity $(\overrightarrow{\mathcal{R}})^{2} \equiv\left(\vec{R}_{(4)}\right)^{2}$ is equal to its low bound which is zero. As follows from formulae (29), (30), the single equation corresponds to all these elements

$$
\frac{d^{4} u}{d t^{4}}=0
$$

The general solution of this equation has the form

$$
u(\tau)=C_{1}+C_{2} \tau+C_{3} \tau^{2}+C_{4} \tau^{3} .
$$

The arbitrary constants $C_{k}, k=\overline{1,4}$, in the control (31) are found with the help of formulas (31), (17), (13) analogously to how it was done in the preceding section. As a result, we obtain

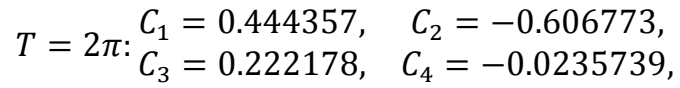

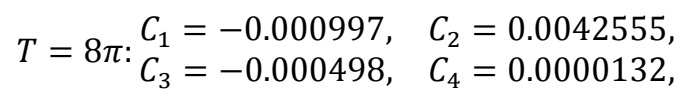

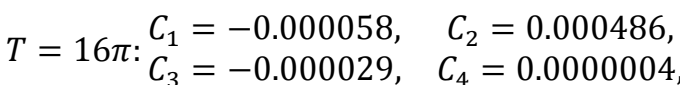

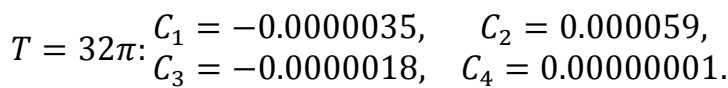

Computational results are presented in Figures 2, 3, 4 and 5 by continuous curves. 

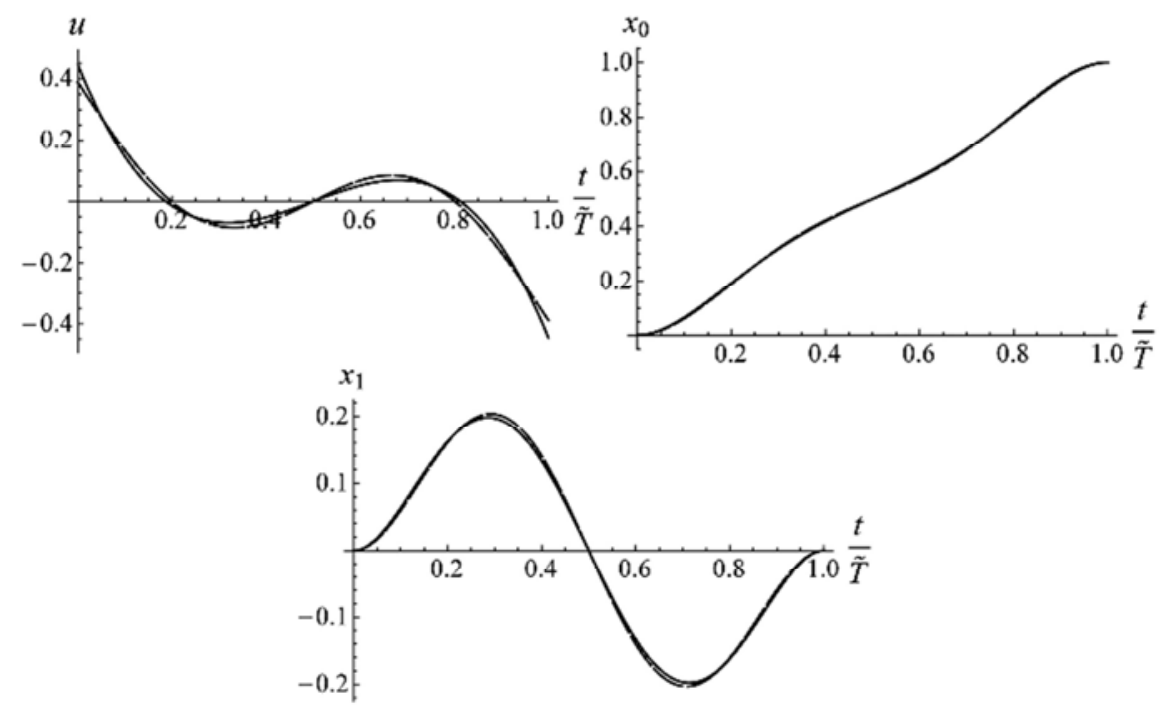

Figure 2. Motion when $T=2 \pi$.
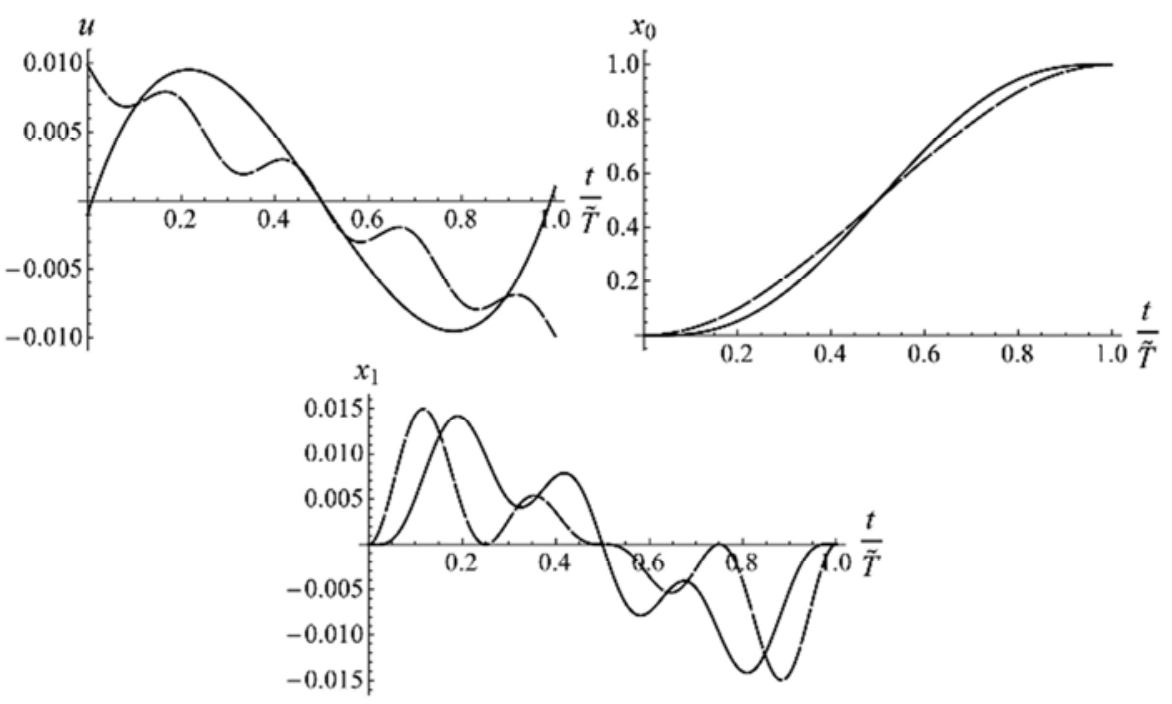

Figure 3. Motion when $T=8 \pi$.
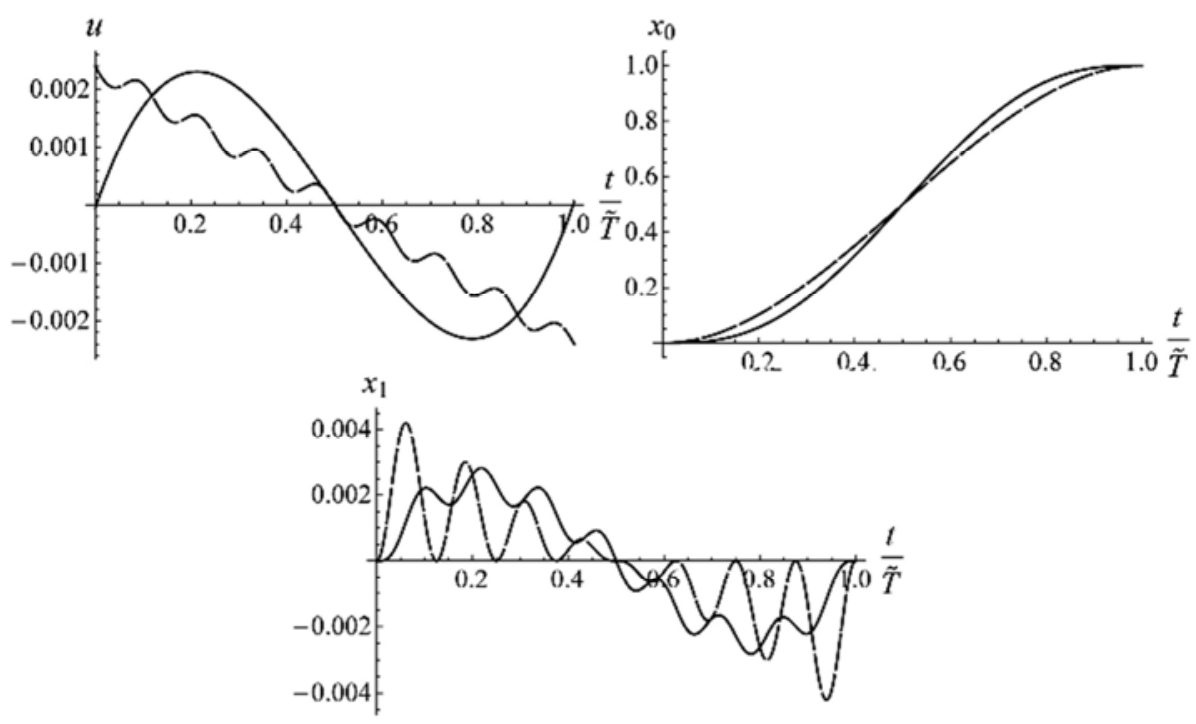

Figure 4. Motion when $T=16 \pi$. 

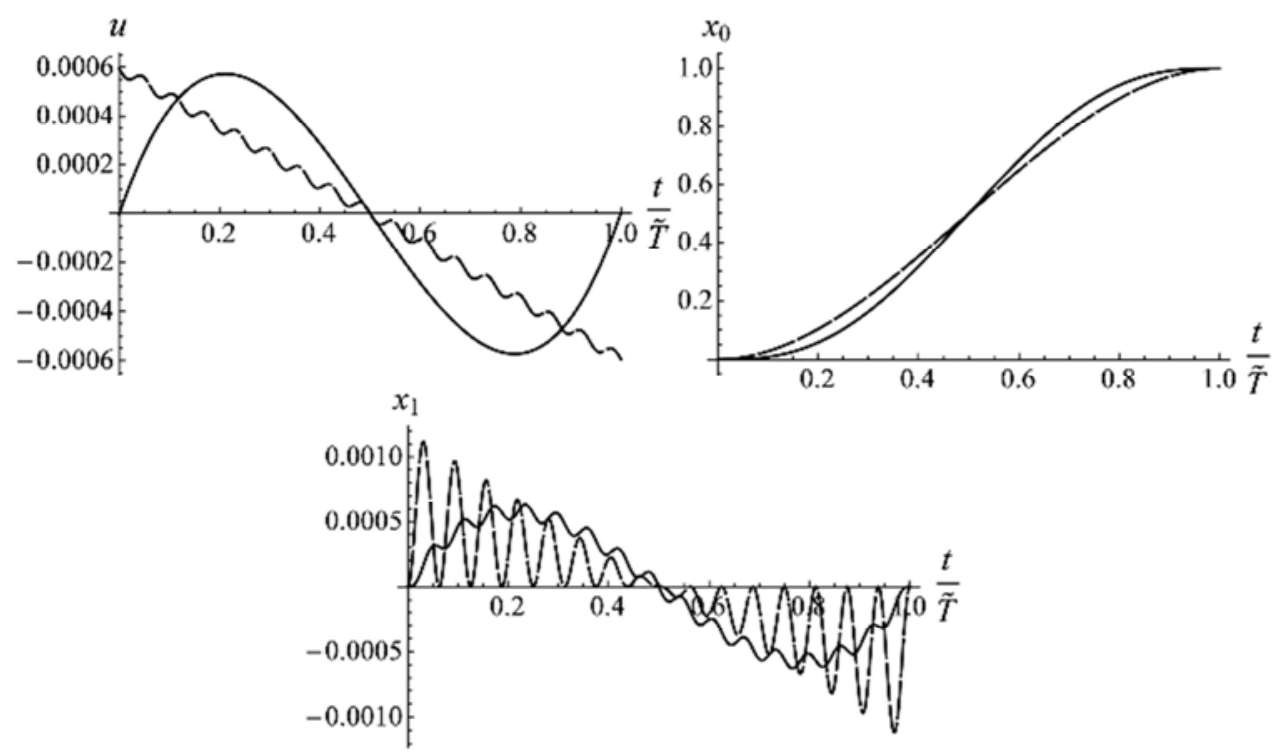

Figure 5. Motion when $T=32 \pi$.

\section{Conclusion}

In the paper the control that transfers a two-mass system with a spring from the initial state of rest to the final state of rest by the given distance in horizontal direction during the time prescribed is found. The problem was solved by the two different principles dealing with the different fields of mechanics - the control theory and the non-holonomic mechanics. In the first case the Pontryagin maximum principle was applied (let us call it the first method), and in the second case the generalized Gauss principle was used (call it the second method).

It is interesting that when the time of motion is short the both methods give practically the same results (see the graphs in Figure 2), but when the time of motion is long the results differ widely (see Figures 3-5). Good agreement in the first case justifies the use of the method from the theory of non-holonomic high-order constraint mechanics for the problems formulated in the control theory, since the results coincide with the values obtained by the classical approach basing on the Pontryagin maximum principle.

If the time of motion is long then the second method is more preferable than the first one, since the control obtained by the second method sways the mechanical system less than the control obtained by the classical approach. This can be explained by the fact that the first method contains the control including harmonics with the natural frequency of the system (remind that the dimensionless natural frequency of the system $\omega_{1}=1$ ), and this seeks to put the system into resonance. In contrast to this, in the second method the control is sought in the form of time polynomial that provides relatively smooth motion of the system.

It is also of interest to note that the first method always finds the control with jumps at the beginning and at the end of motion. The second method also gives the same jumps when the time of motion is short, but when the time of motion is long the similar jumps vanish when one uses the generalized Gauss principle.

\section{Acknowledgments}

The authors express their deep gratitude to Galina Sinilshchikova for the help in preparing and translating the paper into English.

\section{References}

[1] L. S. Pontryagin, V. G. Boltyanskii, R. V. Gamkrelidze, E. F. Mishchenko. The mathematical theory of optimal processes. Translated from Russian by K. N. Trirogoff; edited by L. W. Neustadt, Interscience Publishers John Wiley and Sons, Inc. NewYork-London, 1962. 360 p.

[2] R. Bellman. Dynamic programming. M.: Izd-vo inostr. lit. 1960. 400 p. (in Russian).

[3] N. N. Moiseev, Yu. P. Ivanilov, E. M. Stolyarova. Optimization methods. M.: Nauka. 1978. 352 p. (in Russian).

[4] F. L. Chernous'ko, N. V. Banichuk. Variational problems in mechanics and control. Numerical methods. M.: Nauka. 1973. 238 p. (in Russian).

[5] N. N. Polyakhov, S. A. Zegzhda, M. P. Yushkov. Generalization of the Gauss principle to the case of non-holonomic high-order systems // Doklady AN SSSR. 1983. V. 269. № 6. Pp. 1328-1330 (in Russian).

[6] Sh. Kh. Soltakhanov, M. P. Yushkov, S. A. Zegzhda. Holonomic and non-holonomic constraint forces of any order // Proceedings of the Second International Conference on dynamics, vibration and control. August 23-26, 2006, Beijing, China. P.172.

[7] Sh. Kh. Soltakhanov, M. P. Yushkov, S. A. Zegzhda. Application of the generalized Gaussian principle to the problem of suppression of mechanical systems oscillations // Proceedings of The Third International Conference on Dynamics, Vibration and Control (Hangzhou, China), 2010. CD: File 345.pdf. 
[8] S. A. Zegzhda, P. E. Tovstik, M. P. Yushkov. The Hamilton-Ostrogradski Generalized Principle and Its Application for Damping of Oscillations // ISSN 1028-3358, Doklady Physics, 2012, Vol. 57, No. 11, pp. 447-450. C Pleiades Publishing, Ltd., 2012.

[9] S. A. Zegzhda, E. A. Shatrov, M. P. Yushkov. A new approach to finding the control that transports a system from one phase state to another // ISSN 1063-4541. Vestnik St. Petersburg University. Mathematics, 2016, Vol. 49, No. 2, pp. 183-190. C Allerton Press, Inc., 2016.

[10] S. A. Zegzhda, E. A. Shatrov, M. P. Yushkov. Suppression of oscillation of a trolley with a double pendulum by means of control of its acceleration // Vestnik St. Petersburg University. Ser.1. 2016. Vyp.4. Pp.683-688 (in Russian).
[11] S. A. Zegzhda, Sh. Kh. Soltakhanov. Application of a generalized Gauss principle to solving the problem of suppression of oscillation of mechanical systems // Izv. RAN. Theory and control systems. 2010. №2. Pp.20-25. (in Russian).

[12] F. L. Chernousko, D. Akulenko, B. N. Sokolov. Control of oscillations. Nauka, Moscow, 1980. 384 p. (in Russian).

[13] N. N. Polyakhov, S. A. Zegzhda, M. P. Yushkov. Theoretical Mechanics. M.: Vysshaya shkola, 2000. 592 p. (in Russian).

[14] Sh. Kh. Soltakhanov, S. A. Zegzhda, M. P. Yushkov. Mechanics of non-holonomic systems. A New Class of control systems. Berlin Heidelberg: Springer-Verlag. 2009. 329 p.

[15] B. A. Dubrovin, C. P. Novikov, A. T. Fomenko. Contemporary geometry. M.: Nauka.1979. 760 p. (in Russian). 